and hence Milne's predicted value of $\rho$ is of the order of $10^{-27} \mathrm{gm} . / \mathrm{cm} .^{8}$. On the other hand, in the Einstein universe, which is the basis of Eddington's system, space is hyperspherical; consequently,

$$
M=2 \pi^{2} \rho R^{3} \text {. }
$$

With the value assigned by Eddington to the Einstein radius of space, $\rho$ is again of the order of $10^{-27} \mathrm{gm} . / \mathrm{cm} .^{3}$, but at the present epoch of expansion Eddington considered that the radius is a bout flve times? Einstein radius, giving $\rho$ of the order of $10^{-20} \mathrm{gm} . / \mathrm{cm}^{3}$.

Hence, rivai theories assign numerically different values for the local density distribution. Nevertheless, they agree in isolating mass parameters of the same order of magnitude. As a tentative physical explanation of this theoretical phenomenon, it is suggested that $10^{55} \mathrm{gm}$. is the order of mass which a homogeneous and spherically symmetrical continuous material system must possess if : (i) its gravitational potential energy is comparable with its inertial potential energy ; and (ii) its radius is of the order of $2 \times 10^{\circ}$ light-years.

Department of Mathematies and Mechanics,
Imperial College of Science and Technology,

$$
\text { June } 21 \text {. }
$$

${ }^{1}$ Milne, Proc. Roy. Soc., A, 154, 43 (1936).

2 Milne, Quart. J. Math., 5, 64 (1934).

Milne and McCrea, Quart. J. Math., 5, 73 (1934).

"Heckmann, "Theorien der Kosmologie" (1942)

Milne, Proc. Roy. Soc., A, 154, 43 (1936).

Hubble, "Observational Approach to Cosmology" (1937), 20

7 Eddington, Mon. Not. Roy. Ast. Soc., 104, 203 (1944).

\section{Cascade Showers under Thin Layers of Materials}

IN a previous paper, Bhabha and Chakrabarty ${ }^{1}$ discussed the general theory of the production of cascade showers. The solution obtained there was finally evaluated by the saddle-point method, and it was shown that the same expression cannot be used for small values of $t$ and $y_{0}$, where $t$ is the thickness of the material in radiation units and $y_{0}$ is $\log \left(E_{0} / \beta\right)$, where $E_{0}$ is the energy of the primary particle and $\beta$ is the critical energy for the material. Consequently in that paper numerical values were obtained only for values of $y_{0} \geqslant 3$ and $t \geqslant 2$. It can be seen, however, that $P(E, t) d E$, which represents the number of particles (electrons and positrons) having energies between $E$ and $E+d E$ (produced by a primary particle of energy $E_{0}$ ) at a depth $t$ below the surface of the material, is exactly given by

$P(E, t)=\frac{1}{2 \pi i} \int_{\kappa-i \infty}^{\kappa+i \infty} e^{r t} d r \cdot \frac{1}{2 \pi i E_{0}} \int_{\sigma-i \infty}^{\sigma+i \infty}\left(\frac{E_{0}}{E}\right)^{s}$

$\left\{\sum_{n=0}^{\infty}\left(-\frac{\beta}{E_{0}}\right)^{n} \cdot \frac{\Gamma(s)}{\Gamma(s-n)}{\underset{i=0}{n}}_{i=0}^{D+r} \frac{\left.D+\lambda_{8}-i\right)\left(r+\mu_{8}-i\right)}{(r+1)}\right\} d s$,

where $\lambda_{s}, \mu_{s}$ are functions of 8 (see ref. 1 ). For convenience in calculations, we can express (1) in the form

$$
\begin{gathered}
P(E, t)=\frac{1}{2 \pi i} \int^{x+i \infty} \begin{array}{c}
e^{r t} d r . \\
x-i \infty
\end{array} \\
\frac{1}{2 \pi i E_{0}} \int_{\sigma-i \infty}^{\sigma+i \infty}\left(\frac{E_{0}}{E+\beta g(s, r)}\right)^{s} f(s, r, \beta) d s . \\
\text { where } \\
f(s, r, \beta)=f(s, r)-\frac{\beta}{E_{0}} f_{1}(s, r)+\left(\frac{\beta}{E_{0}}\right)^{2} f_{2}(s, r)- \\
\left(\frac{\beta}{E_{0}}\right)^{3} f_{3}(s, r)+.
\end{gathered}
$$

and $g(8, r)$ is to be so chosen as to make the series (3) rapidly convergent. It can be shown that if we take

$f_{0}(s, r)=\frac{D+r}{\left(r+\lambda_{s}\right)\left(r+\mu_{-}\right)}$, and $g(s, r)=f_{0}(s+1, r)$, then by taking only the first term $f_{0}(8, r)$ in place of $f(g, r, \beta)$ in $(2)$, we get practically the whole of $P(E, t)$; and this is particularly so for smaller values of $t$. We therefore have, to a very high degree of accuracy,

$$
\begin{gathered}
P(E, t)=\frac{1}{2 \pi i} \int^{\varkappa+i \infty} \begin{array}{c}
d r . \\
\varkappa-i \infty
\end{array} \\
\frac{1}{2 \pi i E_{0}} \int_{\sigma \rightarrow i \infty}^{\sigma+i \infty} e^{r t}\left(\frac{E_{0}}{E+\beta g(s, r)}\right)^{s} \cdot \frac{D+r}{\left(r+\lambda_{s}\right)\left(r+\mu_{\delta}\right)} d s .
\end{gathered}
$$

The integral in (4) can be evaluated by the saddle-point method for all values of $t$ and $y_{0}$, and as such is an improvement on the solution given in ref. 1. Hence if $\exp \psi(8, r)$ represents the integrand of (4),

$E_{0} P(E, t)=\frac{1}{2 \pi}\left[\exp \psi\left(s_{0}, r_{0}\right) \cdot\left\{\frac{\partial^{2} \psi}{\partial r^{2}} \cdot \frac{\partial^{2} \psi}{\partial s^{2}}-\left(\frac{\partial^{2} \psi}{\partial r \partial s}\right)^{2}\right\}^{-1 / 2}\right]_{r=s_{0}},$.

$$
\frac{\partial \psi}{\partial r}=0=\frac{\partial \psi}{\partial s}, \text { simultaneously. }
$$

The total number of particles $N(t)$, at any depth $t$ in the showerproducing layer, can be easily obtained from (4). The values of $N(t)$ accompanying table.

VALURS OF $N(t)$ FOR A PARTICLE EXCITED SHOWER

\begin{tabular}{|c|cccccc|}
\hline$y_{0}$ & 1 & 2 & 3 & 4 & 5 & 6 \\
\hline $\begin{array}{c}0 \cdot 2 \\
0.5\end{array}$ & & & & & & \\
\hline & $1 \cdot 12$ & $1 \cdot 20$ & 1.27 & $1 \cdot 34$ & 1.40 & 1.48 \\
\hline
\end{tabular}

Janossy and $\mathrm{Tzu}^{2}$ have suggested that the solution given by Bhabha and Chakrabarty can also be used for smaller values of $t$, provided we retain also the term containing exp $\left(-\mu_{s} t\right)$ occurring there in the for $t=0.5$ and 0.2 are obtained from that expression. For smaller values of $E_{0}$ which are interesting from the practical point of view, the error comes to about 40 per cent for $t=0.5$ and to 100 per cent r more in the case of $t=0 \cdot 2$

With the above solution it is now possible to deduce the energy spectra of the shower particles at all thicknesses and for all energies of the primary particle.

Alibag Observatory,

S. K. Chakrabarty

$$
\text { June } 17 \text {. }
$$

${ }^{1}$ Bhabha and Chakrabarty, Proc. Roy. Soc., A, 181, 267 (1943)

Janossy and Tzu, Nature, 157, 624 (1946).

\section{Turbulent Flow in Alluvium}

SEveral years ago, I put forward ${ }^{1}$ two original equations for the flow of water in alluvium: the first for the wetted perimeter $P$ in terms of the discharge $Q$, the second for the mean velocity $V$ in terms of the hydraulc mean depth $R$ and water surface slope $S$. Both expressions had the curious characteristic that they appeared to be written in general terms

$$
\begin{gathered}
P={ }^{\prime} x^{\prime} Q^{1 / 2} \cdot{ }^{1 / 2} \cdot . \\
V={ }^{\prime} y^{\prime} R^{2 / 3} S^{1 / 3} \quad . \quad . \quad .
\end{gathered}
$$

The equations were empirical and derived from a mass of hydraulic data. Their simplicity gave cause for the hope that eventually a dynamic theory to justify them would be evolved, and basic dimensionally homogeneous equations for the turbulent flow of water in alluvium derived. In this connexion the following analysis is fruitful. From equation (1)

From equation (2)

$$
(R V)^{2}=Q / x^{2}
$$

$$
V^{5}=y^{3}(R V)^{2} S .
$$

Eliminating $(R V)$, which is a proportional to the Reynolds number,

$$
S=x^{2} V^{5} / y^{3} Q ; \quad \text {. . }
$$

or, on introduction of ' $g$ ' the acceleration due to gravity,

$$
S=K V^{5} / g^{2} Q
$$

in which the coefficient is given by the equation

$$
K=g^{2} x^{2} / y^{3} \text {. }
$$

Equation (3a) is dimensionally homogeneous, and the coefficient $K$ must be either a pure number or a pure number multiplied by one or more dimensionless numbers to a very low power, and of insufficient weight to affect the approximate constaney of the coefficient $K$. The omputed value of $K$ is clearly of great moment.

he 1930 assigned a value or 2 ' fro Pun equation (1) fom a more extended range of observations and assigned to $x$ the viven of $2 \cdot 80$. ' ther observations on other collections of data have given values of ' $x$ ' ranging from $2 \cdot 5$ to 3 ' 0 . The Punjab collection is more authoritative. My latest value of ' $y$ ' is $16 \cdot 0$. It wotld not appear possible from observations on canais to obtain values of these coef 2 . If for ' $x$ a value of 2.81 is taken, and for ' $y$ ' a value of 16.0 , ' $g$ ' being given a value of $32 \cdot 2$, the value of $K$ as computed from equation (4) is precisely the round number 2. The question immediately arises whether this is a pure number. The following theorem which $I$ have derived enables If a rrive at a tentative conclusion on this point. metrical alluvial the horizontal and vertical axes of an ideal asymcharacterizing the effective width of the channel, and $D$ a length characterizing the effective depth. The intersection of the axes at the centre of the water surface may be regarded as the polar origin of generation of the channel section by the turbulent water flowing with a discharge of $Q$. There are thus two lengths involved, $\frac{1}{2} W$ and $D$. 